\title{
Neutropenia febril: análisis de los factores pronósticos y el tratamiento adaptado al riesgo. Revisión crítica
}

\author{
A. Carmona Bayonas ${ }^{1}$, J. A. Herrero Martínez ${ }^{2}$, J. Martínez García ${ }^{1}$, M. Marín Vera ${ }^{1}$, \\ M. De las Heras González ${ }^{3}$, A. Navarrete Montoya ${ }^{1}$
}

\section{Resumen}

Los pacientes con neutropenia febril constituyen una población heterogénea. La tendencia actual de la mayoría de los grupos de trabajo consiste en tratar de ofrecer un tratamiento individualizado adaptado al riesgo de complicaciones. El aspecto más crítico se basa en encontrar las variables adecuadas para seleccionar a los enfermos de bajo riesgo. Desafortunadamente, los sistemas pronósticos más utilizados presentan una tasa de errores que se puede considerar inaceptable. Este estudio pretende analizar todos los factores pronósticos implicados en la confección de un algoritmo de tratamiento adaptado al riesgo, que incluya el manejo ambulatorio. Los factores pronósticos evaluados comprenden los sistemas clásicos de Talcott y Mascc, cuya utilidad pronóstica puede resultar controvertida. Además se analizan los fundamentos clínicos y microbiológicos de la neutropenia febril, y se propone la integración de todos estos datos, para conseguir una mayor fiabilidad en la evaluación de los pacientes.

\section{Palabras clave:}

Tratamiento ambulatorio. Neutropenia febril. Talcott. Mascc. Factores pronósticos. Cáncer.

Oncología, 2006; 29 (5):206-218

\footnotetext{
${ }^{1}$ Servicio de Oncología Médica

${ }^{2}$ Servicio de Infecciosas

${ }^{3}$ Servicio de Oncología Radioterápica 


\section{Summary}

Patients with febrile neutropenia are a heterogeneous population. The current tendency of the majority of specialists consists in giving an individual treatment adapted to the risk of complications. The critical aspect is to find suitable variables to select the low risk patients. Unfortunately, the most common prognostic systems have an unacceptable rate of error. The purpose of this study is to analyze all the significant prognostic factors when making an algorithm based on risk factors. The prognostic utility of Talcott and Mascc systems is controversial. Therefore, we propose to integrate the clinical, laboratory and microbiological data in the prognostic schedule.

Key words: Febrile neutropenia. Risk. Prognostic factors. Outpatients. Home treatment. Cancer. Talcott. Mascc.

\section{Introducción}

Los pacientes con fiebre neutropénica presentan un riesgo muy elevado de tener complicaciones graves, y por ello deben ser tratados precozmente con antibióticos. Esta estrategia ha supuesto un éxito considerable en la reducción de las tasas de mortali$\operatorname{dad}^{1}$. En los últimos años asistimos a un cambio de paradigma en el manejo de este síndrome. La tendencia de la mayoría de los grupos de trabajo es establecer parámetros que permitan diseñar estrategias diagnósticas y terapéuticas individualizadas, basadas en la predicción del riesgo, sin olvidar las particularidades epidemiológicas y de la flora bacteriana en cada centro. Recientemente se han publicado varios ensayos randomizados que han sugerido que el tratamiento domiciliario puede ser tan eficaz como el ingreso con antibióticos intravenosos ${ }^{14-16}$. No obstante, los criterios de inclusión de dichos estudios utilizan modificaciones empíricas de los modelos pronósticos de Talcott y Mascc, que tienen tasas de error del 5-6\%. Por tanto, para poder elaborar un algoritmo terapéutico efectivo basado en el riesgo, urge conocer mejor las características del enfermo con neutropenia febril.

Hay varias formas de mejorar los resultados actuales. Los reactantes de fase aguda han demostrado su utilidad como marcadores pronósticos en diversas infecciones. Diversos estudios ${ }^{26}$ sugieren que cuando se emplean criterios clínicos y marcadores de fase aguda de forma conjunta, se evalúa mejor al paciente. Por otra parte no se deben olvidar las bases clínicas y microbiológicas de la fiebre neutropénica, que determinarán la elección del antibiótico más adecuado y pueden recomendar el tratamiento hospitalario.

Finalmente, es preciso conocer mejor las variables pronósticas específicas del episodio tratado en el domicilio, que pueden ser distintas a las consideradas en los modelos actuales. El objetivo final es garantizar que no se somete a los pacientes tratados en la casa a un exceso de riesgo innecesario.

\section{Historia y significación}

Históricamente, Bodey ${ }^{2}$ fue el primero en recoger la evolución de 52 pacientes con leucemia hospitalizados por fiebre neutropénica. Observó que el porcentaje de infecciones comenzaba a aumentar cuando los granulocitos descendían por debajo de $1000 / \mathrm{mm}^{3}$, y que cuando el recuento bajaba de $500 / \mathrm{mm}^{3}$ se incrementaba ostensiblemente el porcentaje de bacteriemias graves. Además, resultó patente que la neutropenia prolongada durante más de una semana, así como la acompañada de leucemia en progresión, tenía un pronóstico claramente peor. Otro factor pronóstico puesto de relieve por Bodey fue la demora en la instauración de tratamiento correcto. De esta forma, la supervivencia en pacientes 
neutropénicos, con bacteriemia por P. aeruginosa ${ }^{3}$, disminuye del $74 \%$ al $46 \%$ cuando el tratamiento específico se demoró más de 24 horas. Schimpff ${ }^{4}$ confirmó estos datos, dentro del estudio que popularizó el concepto de doble cobertura para P. aeruginosa, que aún impera en muchos protocolos. En una época tan reciente como los años 80, Pizzo publicó dos brillantes estudios clínicos ${ }^{5,6}$ que perfilaron el estándar actual, al introducirse dos nuevos conceptos: la adición de antifúngicos si la fiebre no se resuelve, y el mantenimiento de los antibióticos hasta la recuperación medular, para evitar las bacteriemias disruptivas. En 1989 la EORTC confirmó la utilidad de anfotericina B-deoxicolato, en pacientes con fiebre neutropénica mantenida ${ }^{7}$. No obstante, la neutropenia de más de una semana, es un hecho excepcional en una planta de Oncología Médica, por lo que los hongos y las bacteriemias disruptivas, en general no constituyen un problema clínico frecuente.

Por último, los ensayos de la EORTC ${ }^{8}$ establecieron que los glucopéptidos no suelen ser necesarios como terapia inicial, salvo en instituciones con una alta prevalencia de $\mathrm{S}$. viridans resistente a penicilina. Las recomendaciones de la IDSA (Infectious Diseases Society of America $)^{9}$ aconsejan usar vancomicina en infecciones relacionadas con catéteres, colonización por Staphilococo resistente a meticilina, deterioro cardiovascular, o bacteriemia por un gram positivo mientras no llega el antibiograma ${ }^{10}$.

\section{Rasgos del paciente con fiebre neutropénica y un tumor sólido}

La información que disponemos sobre factores pronósticos en pacientes con neoplasias sólidas, a veces está sesgada por la inclusión en los ensayos de enfermos con enfermedades hematológicas, lo que puede originar conclusiones discordantes con la experiencia clínica cotidiana.

El paciente tratado con dosis estándar de quimioterapia suele desarrollar una neutropenia de grado 4 corta, con una duración media de 2-3 días. La fiebre se mantiene durante 1-2 días y por tanto, en el $80 \%$ de las ocasiones no se modifica el esquema antibiótico inicial. Los ingresos son cortos, con una duración media de 4 días, por lo que es común prescribir un tratamiento secuencial oral. En la mayoría de las ocasiones no se encuentra un foco clínico ni se aíslan microorganismos en los cultivos, por tanto, el $40-60 \%$ de los casos se clasifican como fiebre de origen desconocido, y sobre ellos hay muy poca bibliografía referente al mecanismo etiopatogénico subyacente. Aparece un foco clínico evidente desde el principio, en el $25 \%$ de las ocasiones, mientras que los hemocultivos son positivos sólo en aproximadamente un $10 \%$ de los casos. Las muertes infecciosas son inferiores al $2 \%$, y en general se enmarcan en el contexto de enfermos deteriorados previamente por su enfermedad de base.

En contraste, los pacientes con leucemias tienen hemocultivos positivos en el 25-30\% de los $\operatorname{casos}^{11}$, la neutropenia tarda dos semanas en recuperarse ${ }^{12} y$ ello permite la entrada en escena de los hongos y nuevas oleadas de bacteriemias disruptivas ${ }^{13}$.

\section{Sistemas pronósticos. MASCC y Talcott}

El conocimiento de las características de los pacientes con neutropenia febril ha ido incrementándose, de tal forma que ahora se intenta ofrecer a los pacientes un tratamiento individualizado basado en el riesgo. En la última década, se han comunicado las primeras experiencias en este sentido ${ }^{14-16}$, pero en general, no se puede descartar que los pacientes reclutados no hayan sido expuestos a un exceso de riesgo innecesario. Se trata de estudios de tratamiento ambulatorio con escaso tamaño muestral, con criterios de inclusión y esquemas de antibióticos poco comparables, lo que dificulta un análisis crítico correcto, y las elevadas tasas de reingresos y complicaciones pueden resultar inaceptables, en pacientes que en teoría tenían bajo riesgo.

En los últimos años se están utilizando diversos sistemas pronósticos para la estratificación de los episodios, principalmente los de Talcott y MASCC.

\subsection{El sistema de Talcott}

El primer sistema pronóstico se debe a Talcott ${ }^{17}$, que en 1988 publicó una revisión retrospectiva, que incluyó al menos un 50\% de pacientes con tumores hematológicos. El elevado número de pacientes con leucemia, limita su valor pronóstico, porque distorsiona la interpretación de los resultados en pacientes 
con tumores sólidos. Por otra parte, el hecho de que el estudio es una revisión retrospectiva de casos, desembocó en que sólo se definieron 4 grupos pronósticos. Ello se ha planteado falsamente como una ventaja a la hora de su utilización en urgencias, pero en realidad lastra su valor predictivo. Los 4 grupos de Talcott se recogen en la Tabla I. El grupo I comprende los casos que acontecen en mitad de un ingreso por otro motivo. El grupo II incluye la neutropenia febril con algún signo de deterioro clínico desde el principio, es decir, con alguna complicación secundaria al proceso infeccioso, ya establecida. El grupo III principalmente incluye a pacientes con leucemia que no han entrado en remisión completa tras un ciclo de quimioterapia. El grupo IV comprende al resto de pacientes, y es el más numeroso (70\% de los episodios).

TABLA I

\begin{tabular}{|llcc|}
\hline Grupo & Característica & $\begin{array}{c}\text { Tasa de } \\
\text { complicaciones }\end{array}$ & Mortalidad \\
\hline I & Nosocomial & $34 \%$ & $13 \%$ \\
II & Comorbilidad & $55 \%$ & $12 \%$ \\
III & Neoplasia sin control & $31 \%$ & $18 \%$ \\
IV & Ninguna & $2 \%$ & $0 \%$ \\
\hline
\end{tabular}

La validación del estudio encontró que el 5\% de los pacientes considerados de bajo riesgo desarrollaron complicaciones ${ }^{18}$. Un oncólogo dispuesto a ofrecer un alta precoz en función de este sistema se enfrenta con varios problemas. Si el paciente pertenece a los dos primeros grupos, es obvia la necesidad de ingreso: el paciente ya está grave y por tanto hay que ofrecer todas las medidas de soporte. No obstante, salta a la vista que estas dos variables son estadísticamente muy potentes, pero en cambio inefectivas desde un punto de vista práctico, ya que indican una situación de gravedad en la que el único traje a medida debe ser ingresar al enfermo con antibióticos por vía intravenosa. Es posible que si este cajón de sastre se hubiese excluido, otras variables más sutiles como la mucositis hubiesen tenido una consideración diferente.

\subsection{Críticas al sistema de Talcott}

La alta tasa de complicaciones graves del grupo I
(34\%) se debe a la inclusión de pacientes con leucemia que pasan toda la aplasia medular en el hospital, y por tanto es probable que sufran no una, sino varias complicaciones asumidas como parte ineludible del protocolo de quimioterapia, en contraste con la evolución habitual de los enfermos con tumores sólidos.

Respecto al grupo II, en su propia definición lleva implícito un criterio de ingreso y cuidado máximo, por lo cuál, como decimos, su utilidad se ve limitada.

El grupo III, abarca a pacientes con leucemia que no han entrado en remisión completa tras un ciclo de quimioterapia. En pacientes con tumores sólidos es difícil demostrar una progresión intra-tratamiento, y de hecho Talcott clasificó a los enfermos en este estrato utilizando técnicas diagnósticas solicitadas con posterioridad al ingreso por fiebre neutropénica, y ni siquiera está claro qué criterio de progresión se empleó.

Por último, el grupo IV abarca a individuos que seguían un régimen ambulatorio de quimioterapia, y aparentemente no tenían otro problema salvo la fiebre y un hemograma con neutropenia de grado 4, lo cuál abarca a la mayoría de los pacientes. El significado biológico de este estrato reside en que si un paciente sigue estable a pesar de no haber sido tratado, es poco probable, que tras comenzar los antibióticos atraviese complicaciones.

Probablemente, el mérito de los estudios de Talcott resida en haber sugerido que la fiebre neutropénica no es un síndrome homogéneo, y por tanto requiere un tratamiento individualizado. En su estudio, el principal factor que predijo un curso clínico sin complicaciones tras los antibióticos, fue no tenerlas ya antes de los mismos.

\subsection{El sistema MASCC}

El grupo MASCC evaluó a 1531 pacientes pertenecientes a 15 países $^{19}$. Por tanto, se trató de un estudio más grande, de tipo prospectivo, que nuevamente incluyó al menos a un 50\% de pacientes con neoplasias hematológicas. Tras la realización del análisis multivariante quedaron 8 variables como factores de riesgo independiente. Dichas características fueron asociadas a una puntuación, tal y como indica la Tabla II.

El valor máximo es 26 y a más puntos mejor pro- 
TABLA II

\begin{tabular}{|lc|}
\hline Característica & puntos \\
\hline 1. Asintomático o síntomas leves & 5 \\
2. No hipotensión (Tas $>90 \mathrm{~mm} \mathrm{Hg}$ ) & 5 \\
3. No EPOC & 4 \\
4. No infección fúngica previa & 4 \\
5. No deshidratación que requiera fluidoterapia & 3 \\
6. Síntomas moderados & 3 \\
7. Paciente ambulatorio & 3 \\
8. Edad <60 años & 2 \\
\hline
\end{tabular}

nóstico. Una puntuación $\geq 21$ identifica al grupo de bajo riesgo con los siguientes parámetros predictivos: $\mathrm{S}=80 \%, \mathrm{E}=71 \%$, valor predicho positivo $(\mathrm{VPP})=94 \%$, valor predicho negativo $(\mathrm{VPN})=39 \%$. Hay que subrayar el alto VPP que ofrece el sistema. No obstante, el $6 \%$ de pacientes identificados como de bajo riesgo, tuvieron una complicación importante y el $1 \%$ murió, lo cuál puede ser considerado una cifra poco asumible para tomar una decisión crítica. Por último, los pacientes considerados de alto riesgo, tuvieron una tasa de complicaciones del 39\% con un $14 \%$ de mortalidad.

En junio de 2004 se publicó una validación prospectiva del método $\mathrm{MASCC}^{20}$. El $70 \%$ de los pacientes tenían tumores sólidos, pero el resto padecían enfermedades hematológicas. De los 58 pacientes calificados como de bajo riesgo ( $\geq 21$ puntos), sólo 1 tuvo como complicación una infección fúngica, y se recuperó tras el tratamiento. Por el contrario, de los 22 pacientes catalogados como de alto riesgo, el $50 \%$ experimentaron complicaciones, y el $36 \%$ falleció. Llama la atención la mejora de los valores estadísticos de esta validación, frente al estudio original, con un VPP 98.3\%, circunstancia poco frecuente que los autores achacan en parte a la mayor proporción de enfermos con neoplasia sólida. Estos datos son una confirmación del buen pronóstico de la neutropenia febril en pacientes con tumores sólidos.

\subsection{Problemas del sistema MASCC}

Hay que destacar que el punto de corte establecido en 21, surgió bajo la premisa de una asignación arbitraria, que concedía una importancia similar a todos los valores predictivos en liza. No obstante, en la práctica asistencial, las consecuencias de tratar ambulatoriamente a pacientes inestables (falso positivo) pueden ser catastróficas, mientras que ingresar a un exceso de enfermos estables es asumible.

En efecto, si se utiliza como punto de corte 22 puntos, aumenta la tasa de error en la clasificación del 30 al $45 \%$, por una pérdida de especificidad, pero el VPP, que es lo que de verdad importa, asciende del 94 al $97 \%$.

Consideraciones estadísticas aparte, al estudio MASCC se le puede criticar que soslaya el valor del tratamiento de soporte. No sin razón los hematólogos se quejan de que su aplicación estricta desemboca en errores como estratificar de bajo riesgo y por tanto, apto para continuar tratamiento conservador en el ámbito domiciliario, a un paciente sometido a transplante con soporte de progenitores hematopoyéticos. Por el contrario, las bajas cifras de mortalidad del transplante autólogo son debidas al seguimientos casi intensivo. De esta forma, el sistema MASCC no reconoce la multiplicidad de problemas que atraviesa en su evolución un paciente con un tumor, con las barreras mucosas alteradas y con un síndrome mielotóxico global. La anemia o la trombopenia no tienen cabida como predictores de complicaciones, porque el abordaje hospitalario las impide. Si los pacientes no tienen más problemas es porque se les transfunde, se les hidrata, se corrigen alteraciones analíticas leves... y todo ello sólo es posible realizarlo dentro de una infraestructura hospitalaria.

En el estudio MASCC, algunas variables útiles pueden haber quedado eclipsadas, por la enorme fuerza estadística de otras variables como la 2 y la 5 , que no olvidemos son complicaciones ya establecidas, dentro de un sistema pronóstico de las mismas.

Otro aspecto de preocupación lo constituye la introducción de parámetros subjetivos, como son las variables 1 y 6 , referentes a la carga de enfermedad. Ello abre la puerta a la introducción de sesgos, pero además hay que considerar que el control de síntomas forma parte del tratamiento del cáncer, y por tanto es posible que algunos pacientes adscritos al apartado 6 de MASCC deban ser ingresados. El antecedente de infección fúngica (apartado 4) sólo es un factor de riesgo en el paciente hematológico. Por todo ello, el sistema MASCC puede no ser muy útil en pacientes con tumores sólidos.

En cuanto a los pacientes de alto riesgo, MASCC se limita a advertir al oncólogo exactamente lo que 
TABLA III

\begin{tabular}{|c|c|c|}
\hline Autor & Marcador & Conclusión \\
\hline Manian $1995^{21}$ & PCR & $\begin{array}{l}\text { Elevación } \geq 50 \text { mg/L D1-2 distingue bacteriemia de infección localizada con un VPP } \\
95 \%\end{array}$ \\
\hline Santolaya $1994^{22}$ & PCR & $\begin{array}{l}\text { PCR } \geq 40 \mathrm{mg} / \mathrm{L} \text { detecta una infección bacteriana, con sensibilidad del } 100 \% \text { y especifi- } \\
\text { cidad del } 76.6 \% \text {. }\end{array}$ \\
\hline Engervall $1995^{23}$ & PCR / IL 6 & $\begin{array}{l}\text { IL- } 6>100 \mathrm{ng} / \mathrm{L} \text { en los días } 3-5 \text { se asoció a fiebre }>7 \text { días. Il-6 }>100 \mathrm{ng} / \mathrm{L} \text { tiene una sen- } \\
\text { sibilidad del } 66 \% \text { para detectar bacteriemia por gram negativo y del } 22 \% \text { para detectar } \\
\text { bacteriemia por gram positivo. }\end{array}$ \\
\hline De Bont $1999^{24}$ & IL 6 / IL 8/ PCR & $\begin{array}{l}\text { La elevación de Il- } 6 \text { e Il-8 distingue entre bacteriemia y fiebre de origen desconocido. } \\
\text { Es posible discriminar entre bacteriemias por gram positivos y gram negativos. }\end{array}$ \\
\hline Persson $2004^{25}$ & $\begin{array}{l}\text { Procalcitonina, } \\
\text { IL 6/ IL 8, PCR }\end{array}$ & $\begin{array}{l}\text { Marcadores son útiles para descartar bacteriemia. Los mejores valores predictivos se } \\
\text { obtuvieron con procalcitonina (VPP } 67 \% \text {, VPN } 94 \% \text { ). }\end{array}$ \\
\hline Giamarellou $2004^{26}$ & Procalcitonina & $\begin{array}{l}\text { Procalcitonina } \geq 5 \mathrm{ng} / \mathrm{ml} \text { tiene una sensibilidad y especificidad del } 83.3 \text { y } 100 \% \text { para } \\
\text { detectar una sepsis. Procacitonina } ₫ 0.5 \mathrm{ng} / \mathrm{ml} \text { indica que la infección es improbable, } \\
\text { (falsos negativos por estafilococos coagulasa negativos). }\end{array}$ \\
\hline Jimeno $2004^{27}$ & Procalcitonina & $\begin{array}{l}\text { Procalcitonina }>0.5 \mathrm{mg} / \mathrm{ml} \text {, tiene una sensibilidad del } 66.7 \% \text { para detectar una infec- } \\
\text { ción microbiana y se asocia a fallo de tratamiento en el } 70 \% \text { de los casos versus } \\
14 \% \text {.Los } 5 \text { pacientes que fallecieron en este estudio tuvieron cifras de procalcitonina } \\
5-10 \text { veces superiores al valor normal. }\end{array}$ \\
\hline
\end{tabular}

ya sabía: si el paciente llegó a urgencias con hipotensión o fallo renal, entonces tiene más posibilidades de seguir teniendo complicaciones que si faltan estos datos. En este sentido hay que tener clara la diferencia entre predecir el curso clínico de un paciente o simplemente confirmarlo.

\subsection{Problemas metodológicos para construir un algoritmo terapéutico}

Los dos métodos descritos son interesantes en tanto que aportan un mayor conocimiento del paciente con neutropenia febril, pero por desgracia hay varios aspectos metodológicos que dificultan su utilización en ensayos de tratamiento ambulatorio. Una objeción es que no fueron desarrollados ex profeso para esa finalidad, por lo que es posible que se necesiten variables específicas distintas.

Por otro lado, ambos sistemas se han construido sobre la base de tratar de identificar a un grupo de pacientes de alto riesgo que puede ser demasiado heterogéneo. Por ejemplo, según el trabajo original de Talcott, el $25 \%$ de las complicaciones tuvieron una causa infecciosa, el $27 \%$ se debió a un problema no infeccioso y al menos en la mitad de los episodios fue difícil discernir cuál era mecanismo etiopatogénico subyacente. Algunas variables pronósticas pueden no haber recibido la importancia que merecían, debido a la falta de homogeneidad de la muestra. Todos estos factores determinan que la tasa de error de las clasificaciones sea muy alta (5-6\% de falsos negativos), lo que resulta inaceptable para tomar decisiones críticas.

\section{Evaluación de marcadores de fase aguda}

Los reactantes de fase aguda son un grupo heterogéneo de proteínas sintetizadas principalmente en el hígado, en respuesta a la inflamación y la necrosis tisular, por lo que han demostrado su utilidad como marcadores pronósticos en diversas infecciones. Los sistemas de estratificación de Talcott y Mascc no consideran ninguno de los marcadores de fase aguda como criterios pronósticos. No obstante, diversos estudios recientes han analizado el papel de las citocinas y otros reactantes de fase aguda, tanto en la predicción de infecciones bacterianas subyacentes, como de resultado clínico. En esta revisión nos centraremos en los más estudiados, como son la PCR, procalcitonina, interleucina-6 e interleucina-8.

Desde comienzos de los 90, se conoce la implicación de determinadas citocinas en la fisiopatogenia de la fiebre neutropénica ${ }^{21}$. Puesto que parece probable que Il-6 e Il-8 sean sintetizados por el endotelio, y no por neutrófilos y monocitos, su papel en la sepsis neutropénica ha sido extensamente estudiado ${ }^{21}$. 
La procalcitonina (PCT) es un péptido de 116 aminoácidos, producido por las células $\mathrm{C}$ del tiroides. En sujetos normales, se transforma rápidamente en calcitonina, por lo que sus niveles en sangre son muy bajos $(0.1 \mathrm{ng} / \mathrm{ml})$. Tras la administración de endotoxina, sus niveles comienzan a subir en 2-3 horas, tienen un pico en 6-8 horas y alcanzan una meseta a las 24 horas.

Probablemente, el enfoque más prometedor para la clasificación pronóstica de la neutropenia febril consista en la elaboración de un modelo que integre variables clínicas y analíticas. En la Tabla III se presenta un resumen de los principales estudios sobre marcadores de fase aguda en la neutropenia febril.

\section{Bases clínicas y microbiológicas}

Para ofrecer un tratamiento efectivo basado en factores pronósticos, es necesario considerar las bases clínicas o microbiológicas de la neutropenia febril. Dichos fundamentos son imprescindibles para guiar la elección de antibiótico ${ }^{28}$, y por tanto pueden condicionar que el paciente pueda o no ser tratado fuera del hospital. En este sentido, es de especial relevancia el significado del uso previo de antibióticos como factor de riesgo y pronóstico de las infecciones. Por ejemplo, el uso previo de betalactámicos con limitada actividad frente a S. pneumoniae se asocia a cepas resistentes a penicilina, o Haemophilus influenzae productor de betalactamasas; la utilización anterior de quinolonas se asocia a infecciones urinarias por E. coli o P. aeruginosa, resistentes a ciprofloxacino; el empleo de levofloxacino se ha relacionado con la emergencia de cepas resistentes de $\mathrm{S}$. pneumoniae, de $\mathrm{P}$. aeruginosa y $\mathrm{S}$. aureus resistente a meticilina. Por último, el uso previo de cefalosporinas de $3^{\text {a }}$ generación se asocia a infecciones por microorganismos gram negativos productores de betalactamasas de espectro ampliado, E. faecalis, S. epidermidis y S. pneumoniae resistente a penicilina ${ }^{29}$.

Puesto que el consumo de antibióticos es muy frecuente en los enfermos con cáncer, este dato podría ayudar a seleccionar pacientes en los que va a fracasar el tratamiento oral. Dichas consideraciones también se aplican a los pacientes con fiebre neutropénica, que han recibido profilaxis antibiótica. Esta estrategia reduce el número de episodios febriles y la incidencia de infecciones documentadas micro- biológicamente, pero de forma intrigante, ello no se traduce en una disminución de la mortalidad específica $^{30}$. Una explicación podría ser la falta de poder estadístico, por el bajo número de fallecimientos en ambos grupos. No obstante, no se puede descartar que los episodios febriles que surgen en el contexto de un tratamiento profiláctico, tengan eventualmente un curso más complicado. En este sentido, se desconoce el rendimiento de los antibióticos de rescate, en pacientes que han recibido profilaxis.

La cuestión se vuelve a suscitar en un ensayo británico publicado recientemente ${ }^{31}$, que aleatoriza a 1565 pacientes en tratamiento con quimioterapia, a recibir de forma profiláctica levofloxacino versus placebo. Efectivamente, se constató una disminución significativa en la tasa de neutropenias febriles y hospitalizaciones. Además se observó una tendencia a la disminución de hemocultivos positivos (6.2\% versus $7.9 \%$ ) y sepsis graves (8 versus 16 pacientes), en el brazo de levofloxacino. Es interesante destacar que en el subgrupo de pacientes sépticos, el pronóstico fue peor si habían recibido profilaxis previa (4/8 frente a 4/16 fallecimientos), aunque los eventos fueron escasos para arrojar conclusiones definitivas. Por ello, no se puede descartar que la profilaxis tuviera un impacto negativo en el rendimiento del tratamiento de rescate posterior.

En definitiva, todas las circunstancias capaces de provocar un cambio en la flora bacteriana endógena, como serían el consumo de esteroides y el ingreso reciente, (muy frecuentes en los enfermos con cáncer), podrían constituir importantes factores pronósticos a tener en cuenta.

Otro apartado interesante es la valoración del foco infeccioso. Los ensayos clínicos con frecuencia no incluyen un número suficiente de pacientes para hacer un análisis de subgrupos a este respecto. Además, las infecciones en el paciente con neutropenia febril se caracterizan por la escasez de hallazgos clínicos, debido a la incapacidad de generar una respuesta inflamatoria adecuada. Por otra parte, en el paciente neutropénico la bacteriemia puede ser resultado de la entrada en el torrente sanguíneo de flora endógena procedente del tracto gastrointestinal, generalmente sin una infección evidente de tejidos. En caso de existir un foco clínico asociado, éste puede considerarse el resultado de una diseminación hematógena de gérmenes saprófitos, más que el origen primario de la bacteriemia. Debido a ello, 
algunos estudios ${ }^{32,33}$ sugieren que es el foco infeccioso más que la propia bacteriemia, lo que confiere un mal pronóstico en el paciente neutropénico, ya que constituye un marcador de extensión y agresividad del microorganismo causal. Sin embargo, no hay información de subgrupos referente a infecciones poco extensas (blefaritis, gingivitis, faringitis leves...), aunque es posible que su pronóstico sea similar a la fiebre de origen oculto, al no reflejar el resultado de una extensión hematógena.

Talcott ${ }^{34}$ concluyó que la bacteriemia se asociaba a un peor pronóstico en todos los grupos excepto el 4. Diversos ensayos clínicos ${ }^{35}$ han registrado una menor tasa de éxito terapéutico sin modificación del esquema inicial en los pacientes con bacteriemia. No obstante, no existe unanimidad respecto al rendimiento del tratamiento correcto en pacientes con y sin bacteriemia. No obstante, es posible que este rendimiento sí disminuya en el domicilio, donde el cumplimiento terapéutico es más difícil de asegurar. La mayoría de los estudios de tratamiento oral han excluido a los pacientes con infecciones clínicas localizadas $^{36}$, por lo que no existen datos pronósticos sobre esta población. Por tanto, una buena forma de avanzar podría ser perfeccionar un método predictivo que discriminara si el paciente que se va enviar a casa tiene una infección bacteriana o no.

Un parámetro que tiene correlación con la tasa de bacteriemia es la profundidad de la neutropenia. En el estudio de Talcott constituyó un dato desfavorable en los grupos I-III pero no en el IV. En cambio, en el estudio MASCC la neutropenia de grado IV se asoció a un peor pronóstico de forma estadísticamente significativa, aunque no resultó ser un factor de riesgo independiente en el análisis multivariante. Algunos ensayos de tratamiento ambulatorio con una alto porcentaje de pacientes con un nadir $<100$ neutrófilos $/ \mathrm{mm} 3$ han comunicado una tasa inaceptable de pacientes reingresados con bacteriemias gra$\operatorname{ves}^{37}$. Es decir, en una situación en la que no se pueda garantizar el cumplimiento terapéutico, es posible que la profundidad de la neutropenia sea un dato a tener más en cuenta.

Uno de los principales factores para mejorar la eficacia terapéutica de los tratamientos consiste en el conocimiento de los microorganismos más frecuentes en cada comunidad. Ello permite seleccionar de forma empírica los antibióticos con una mayor tasa de sensibilidad. Desafortunadamente, algunos ensa- yos con tratamiento ambulatorio no comunican el mapa microbiológico de los lugares donde se llevaron a cabo. Ello limita mucho la extrapolación de resultados a hospitales con un perfil de resistencias diferente. Por tanto, los centros que en el futuro ofrezcan tratamientos ambulatorios, deben asegurar que se efectúan análisis epidemiológicos periódicos, y no se puede descartar que en algunos hospitales la única opción terapéutica en un momento determinado sea el tratamiento con antibióticos intravenosos.

\subsection{La mucositis como factor de riesgo}

La mucositis no se considera una variable pronóstica en el método MASCC o Talcott. Sin embargo, la disrupción de barreras ha sido implicada numerosas veces como factor de riesgo de bacteriemias $^{38}$ y sepsis graves ${ }^{39,40}$. Desgraciadamente, el estudio del grupo MASCC excluyó de forma arbitraria a la mucositis como variable a estudio. Los trabajos de Talcott la relacionan enigmáticamente con un descenso en el riesgo de complicaciones. Una interpretación posible es que, en ciertos pacientes, la mucositis y el síndrome febril asociado a neutropenia sean una consecuencia común de una infección vírica ${ }^{41}$, pero no hay muchos datos que sustenten esta hipótesis. Por otra parte, quizá la controversia resida en la dificultad para valorar de forma objetiva el estómago y el colon, que probablemente tengan más importancia que la boca.

Recientemente, un ensayo aleatorio con palifer$\min ^{42}$ (factor de crecimiento de queratinocitos) versus placebo, ha reavivado el interés por el tema de la mucositis, ya que se observó una correlación entre el descenso en la tasa de mucositis de grado 4, y la aparición de neutropenia febril (75 versus 92\%, $\mathrm{p}<0.05)$, con una tendencia a la disminución en la tasa de bacteriemias.

\subsection{La inmunosupresión como factor de riesgo}

El efecto de la quimioterapia sobre el tejido linfoide es un tema complejo y poco explorado. No obstante, se sabe que el deterioro de la función de los linfocitos T tiene gran repercusión clínica en pacientes con leucemia ${ }^{43}$, debido a la alta prevalencia de reactivaciones víricas ${ }^{44-47}$. La toxicidad asociada a quimioterapia es el principal factor responsable de este deterioro inmunológico ${ }^{48}$. 
Este fenómeno también ha sido documentado en pacientes con tumores sólidos sometidos a quimioterapia estándar ${ }^{49}$. Se ha observado una disminución de linfocitos $\mathrm{T}$ en pacientes tratadas con CMF como quimioterapia adyuvante ${ }^{50}$. La fludarabina predispone a ciertas infecciones oportunistas ${ }^{51}$ y los taxanos pueden producir linfopenias severas y asociarse a infecciones no neutropénicas, independientemente del uso de esteroides ${ }^{52}$. Algunas comunicaciones han implicado a C. albicans y VHS en la mitad de los casos de mucositis y fiebre neutropénica ${ }^{53}$, aunque una relación causal es especulativa. Por otra parte, también se ha observado el efecto contrario. Algunos trabajos sugieren que irinotecan ${ }^{54}$ o la combinación de doxorrubicina y paclitaxel alivian la inmunosupresión secundaria a neoplasias de colon o mama, respectivamente.

A pesar de todo, persiste la creencia de que la inmunosupresión es un fenómeno transitorio y poco relevante clínicamente, en la neutropenia febril de pacientes con tumores sólidos. No obstante, es posible que el deterioro inmunológico asociado a reactivación vírica, pudiera ser un hecho interesante como hipótesis etiológica en un subgrupo de pacientes, lo que permitiría ofertar un tratamiento individualizado.

\section{Factores pronósticos en ensayos de tratamiento ambulatorio}

Diversos ensayos han concluido que el tratamiento con antibióticos orales es factible y efectivo en pacientes considerados de bajo riesgo, y sin infecciones extensas. En dicha población no se han encontrado subgrupos en los que el tratamiento intravenoso sea superior ${ }^{55}$. No obstante, el principal problema del tratamiento oral es la intolerancia gastrointestinal ${ }^{56}$, que puede hacer necesario el ingreso de algunos pacientes. Además, algunos ensayos han relacionado esta modalidad con un aumento en el riesgo de deterioro de la función renal ${ }^{57,58}$, quizá por el menor consumo de agua.

Los resultados favorables alentaron las primeras experiencias de tratamiento ambulatorio. Los primeros trabajos prospectivos, no aleatorios, se realizaron durante la primera mitad de los 90, y concluyeron que el tratamiento ambulatorio era efectivo, bien tolerado y por tanto constituía una buena alternativa al ingreso. Lamentablemente, es posible cuestionar si las tasas de reingreso y de complicaciones eran realmente asumibles $(13-21 \%)^{14,15,59}$. Por otra parte, no se avanzó mucho en el conocimiento de los factores pronósticos específicos del tratamiento ambulatorio, debido al bajo número de pacientes incluidos en los estudios.

Debido a ello, los criterios de inclusión de los ensayos randomizados efectuados con posterioridad, eran empíricos y poco homogéneos ${ }^{60}$, lo que dificulta la interpretación de los datos. En general, se utilizó la clasificación de Talcott con algunas modificaciones intuitivas como excluir a pacientes con neumonía o celulitis extensas. Algunos trabajos excluyeron también a los enfermos que habían tomado antibióticos previos ${ }^{16,61}$ y el ensayo de Mullen ${ }^{62}$ excluyó los episodios asociados a mucositis grave.

El estudio de Hidalgo ${ }^{63}$ quizá sea el más representativo, ya que únicamente incluyó a pacientes con neoplasias sólidas, comunicó el perfil microbiológico, y el brazo control se puede considerar estándar (ceftazidima y amikacina). Si bien no hubo diferencias significativas entre los dos brazos del estudio, fue precisa una revisión del tratamiento en el $20 \%$ de los pacientes tratados de forma ambulatoria.

Todos estos estudios concluyeron que no había diferencias significativas en eficacia, entre el tratamiento ambulatorio y la rama control. Sin embargo, ninguno de ellos contó con una potencia estadística suficiente para efectuar análisis de subgrupos (la muestra más numerosa fue la de Innes ${ }^{16}$ con 102 pacientes).

En general, los motivos por los que se tuvo que reingresar a los pacientes fueron la intolerancia a los fármacos orales, la fiebre persistente con o sin deterioro clínico, y consideraciones microbiológicas tras analizar el resultado de los cultivos.

Es interesante resaltar que los factores pronósticos pueden variar en función de que el episodio se maneje en el hospital o en el domicilio. De esta forma, el trabajo de Escalante ${ }^{64}$ encontró que las únicas variables asociadas al fracaso del tratamiento ambulatorio eran: edad $\geq 70$ años, $\mathrm{PS} \geq 2$, mucositis $>$ grado 2 y neutrófilos $<100$. Notablemente, la edad avanzada es la única variable común con el sistema Mascc.

\section{Conclusiones}

El ingreso y la administración de antibióticos por vía intravenosa, constituye el estándar aceptado, pa- 
ra el tratamiento de la neutropenia febril secundaria a quimioterapia ${ }^{9}$, aunque la vía oral puede ser una alternativa válida en un grupo seleccionado de pacientes, considerados de bajo riesgo ${ }^{55}$. En los últimos años, ha aumentado el conocimiento que tenemos sobre la evolución y factores pronósticos de estos pacientes. Por ello la tendencia actual de los diferentes grupos ha sido tratar de ofrecer a los pacientes un tratamiento individualizado basado en la estimación de riesgo.

Recientemente se han publicado varios ensayos aleatorios que han comparado el tratamiento oral en régimen ambulatorio, versus el tratamiento estándar en el hospital. En estos trabajos no se encontraron diferencias significativas respecto a tasas de eficacia y seguridad ${ }^{16}$. No obstante, existe alguna evidencia de que las variables predictivas importantes en el domicilio pueden ser diferentes a las que se utilizan en el hospital ${ }^{64}$. El número de pacientes reclutados ha sido bajo, por lo que es difícil efectuar análisis de subgrupos. Además, los criterios de inclusión no han sido homogéneos, debido a la controversia existente en torno a los métodos pronósticos, y por ello, es difícil extraer conclusiones definitivas sobre su verdadera significación.

Los dos sistemas pronósticos más estudiados son los de Talcott $^{17}$ y MASCC ${ }^{19}$. Ambos presentan aspectos controvertidos en su metodología, que impiden una correcta valoración de ciertos factores de riesgo acreditados con anterioridad. Así, se han puesto sobre la mesa variables con poca entidad pronóstica (por ejemplo, la hipotensión o la deshidratación en el sistema Mascc, que son complicaciones establecidas en un sistema predictivo de las mismas), por el hecho de que son estadísticamente muy potentes. Además de ello, se ha tratado de caracterizar a una población demasiado heterogénea, con complicaciones no siempre infecciosas (progresión tumoral, hemorragias, arritmias...) y se ha incluido a demasiados pacientes con leucemia, lo cuál distorsiona el análisis de los resultados. Es posible que estas consideraciones hayan permitido el eclipse estadístico de algunos rasgos que de verdad podrían ayudar a distinguir en urgencias, al paciente que va a seguir estable del que no. Los estudios validatorios de ambos sistemas, han encontrado tasas de falsos negativos (5-6\%), que pueden ser consideradas inaceptables para tomar decisiones críticas.

Es posible mejorar los algoritmos de manejo de la neutropenia febril de varias maneras. Una de ellas consiste en integrar los marcadores de fase aguda junto a los criterios clínicos. Diversos estudios han demostrado que valores elevados de procalcitonina podrían ayudar a diferenciar un episodio de bacteriemia de una infección localizada ${ }^{23}$, y seleccionar a pacientes en los que va a fracasar el tratamiento ${ }^{29}$. Por el contrario, niveles muy bajos de procalcitonina podrían indicar que una infección bacteriana es improbable ${ }^{28}$. Las interleucinas 6 y 8, y la PCR también han demostrado extensamente su eficacia pronóstica ${ }^{25,26}$. Por ello, creemos que es factible construir un sistema de estratificación mixto que incluya parámetros clínicos y analíticos, lo que podría mejorar el 5-6\% de falsos negativos de las clasificaciones actuales.

Por otra parte, para ofrecer un tratamiento efectivo, es preciso considerar las bases clínicas y microbiológicas de la neutropenia febril. Dichos fundamentos determinarán la elección del antibiótico idóneo ${ }^{29}$ y por tanto pueden condicionar que el paciente sólo pueda ser tratado en el hospital, independientemente del riesgo estimado. Para ello, es imprescindible tener en cuenta todos los factores involucrados en el desarrollo de resistencias a antibióticos. Es muy razonable no tratar ambulatoriamente a pacientes que hayan recibido esquemas de quimioterapia en infusión continua en régimen hospitalario, porque pueden haber sido colonizados por cepas resistentes. De la misma forma, los enfermos que han recibido antibióticos previos pueden haber desarrollado resistencias, que aconsejen el ingreso y tratamiento intravenoso. Tampoco se ha demostrado que el rendimiento del tratamiento de rescate sea similar en pacientes que han recibido profilaxis. Un ensayo reciente $^{31}$, ha demostrado que levofloxacino administrado durante el nadir de la neutropenia, reduce de forma significativa la tasa de episodios febriles e infecciones ( $34.2 \%$ versus $41.5 \%, \mathrm{p}=0.004)$, frente a placebo. Sin embargo, el número de pacientes con sepsis graves y fallecimientos fue similar en ambos grupos, lo que sugiere que los episodios febriles fueron más graves en los enfermos que recibieron levofloxacino. Por ello, uno de los aspectos que será preciso abordar en futuros ensayos clínicos, es el impacto de la profilaxis en la eficacia del tratamiento de rescate.

Finalmente, es muy probable que algunos pacientes se beneficien de profundizar en el mecanismo 
etiopatogénico de los episodios catalogados como fiebre de origen desconocido.

La integración racional de todos estos conocimientos debe permitir en el futuro, la confección de algoritmos eficaces de tratamiento adaptado al riesgo. Desafortunadamente, la ausencia de camas y los criterios económicos no constituyen un criterio científico añadido. Por todo ello, la mayoría de oncólogos no considera todavía el tratamiento ambulatorio, como el nuevo estándar para el manejo de la neutropenia febril de bajo riesgo, y sólo se recomienda en el contexto de ensayos clínicos.

Correspondencia:

Dr. A. Carmona

trebla_albert@hotmail.com

\section{Bibliografía}

1. Pizzo PA, Robichaud KJ, Gill FA et al. Empiric antibiotic and antifungal therapy for cancer patients with prolonged fever and granulocytopenia. Am J Med 1982;72:101-111.

2. Bodey GP, Buckley M, Sathe YS, Freireich EJ. Quantitative relationships between circulating leukocytes and infection in patients with acute leukemia. Ann Intern Med 1966;64:328-340.

3. Bodey GP, Jadeja L, Elting L.Pseudomonas bacteremia. Retrospective analysis of 410 episodes. Arch Intern Med. 1985 Sep;145(9):1621-9.

4. Schimpff S, Satterlee W, Young VM et al. Empiric therapy with carbenicillin and gentamicin for febrile patients with cancer and granulocytopenia. N Engl J Med 1971;284:1061-1065.

5. Pizzo PA, Robichaud KJ, Gill FA et al. Duration of empiric antibiotic therapy in granulocytopenic patients with cancer. Am J Med 1979;67:194-200.

6. Pizzo PA, Robichaud KJ, Gill FA et al. Empiric antibiotic and antifungal therapy for cancer patients with prolonged fever and granulocytopenia. Am J Med 1982;72:101-111.

7. EORTC International Antimicrobial Therapy Cooperative Group. Empiric antifungal therapy in febrile granulocytopenic patients. Am J Med 1989;86:668-672.

8. Vancomycin added to empirical combination antibiotic therapy for fever in granulocytopenic cancer patients. European Organization for Research and Treatment of Cancer (EORTC) International Antimicrobial Therapy Cooperative Group and the National Cancer Institute of Canada-Clinical Trials Group. J Infect Dis. 1991 May;163(5):951-8. Erratum in: J Infect Dis 1991 Oct;164(4):832.
9. Rolston KV. The Infectious Diseases Society of America 2002 guidelines for the use of antimicrobial agents in patients with cancer and neutropenia: salient features and comments. Clin Infect Dis. 2004 Jul 15;39 Suppl 1:S44-8.

10. Vancomycin does not benefit persistently febrile neutropenic people with cancer. Wade JC, Glasmacher A. Cancer Treat Rev. 2004 Feb;30(1):119-26.

11. Philip A. Pizzo. Management of Fever in Patients with Cancer and Treatment-Induced Neutropenia. NEJM May 6, 1993 Volume 328:1323-1332

12. Leong DC, Kinlay S, Ackland S, Bonaventura A, Stewart JF. Low-risk febrile neutropenia in a medical oncology unit. Aust N Z J Med. 1997 Aug;27(4):403-7.

13. Pizzo PA. After empiric therapy: what to do until the granulocyte comes back. Rev Infect Dis 1987;9:214-219.

14. Malik IA, Khan WA, Karim M et al. Feasibility of outpatient management of fever in cancer patients with low-risk neutropenia: results of a prospective randomized trial. Am J Med 1995;98:224-231

15. Gardembas PM, Desablens B, Sensebe L et al. Home treatment of febrile neutropenia: an empirical oral antibiotic regimen. Ann Oncol 1991;2:485-487.

16. Innes HE, Smith DB, O'Reilly SM et al. Oral antibiotics with early hospital discharge compared with in-patient intravenous antibiotics for low-risk febrile neutropenia in patients with cancer: a prospective randomised controlled single centre study. British Journal of Cancer 2003; 89: 4349.

17. Talcott JA, Finberg R, Mayer RJ et al. The medical course of cancer patients with fever and neutropenia. Clinical identification of a low-risk subgroup at presentation. Arch Intern Med 1988;148:2561-2568.

18. Talcott JA, Siegel RD, Finberg R et al. Risk assessment in cancer patients with fever and neutropenia: a prospective, two-center validation of a prediction rule. J Clin Oncol 1992;10:316-322.

19. Jean Klastersky, Marianne Paesmans, Bernardo Rapoport, Kenneth Rolston, James Talcott. The Multinational Association for Supportive Care in Cancer Risk Index: A Multinational Scoring System for Identifying Low-Risk Febrile Neutropenic Cancer Patients. Journal of Clinical Oncology, Vol 18, Issue 16 (August), 2000: 3038-3051.

20. Almarie Uys, Bernardo Rapoport, Roanald Anderson. Febrile neutropenia: a prospective study to validate the Multinational Association of Supportive Care of Cancer (MASCC) risk-index score. Support Care Cancer 2004; 12:555-560

21. Manian FA. A prospective study of daily measurement of C-reactive protein in serum of adults with neutropenia. Clin Infect Dis. 1995 Jul;21(1):114-21.

22. Santolaya ME, Cofre J, Beresi V. C-reactive protein: a valuable aid for the management of febrile children with cancer and neutropenia. Clin Infect Dis. 1994 Apr;18(4): 58995.

23. Engervall P, Granstrom M, Andersson B, Bjorkholm M.Monitoring of endotoxin, interleukin-6 and C-reactive 
protein serum concentrations in neutropenic patients with fever. Eur J Haematol. 1995 Apr;54(4):226-34.

24. de Bont ES, Vellenga E, Swaanenburg JC, Fidler V, Visser-van Brummen PJ. Plasma IL-8 and IL-6 levels can be used to define a group with low risk of septicaemia among cancer patients with fever and neutropenia. Kamps WABritish Journal of Haematology. 107(2):375-80, 1999 Nov.

25. Persson L, Engervall P, Magnuson A, Vikerfors T, Soderquist B, Hansson LO, Tidefelt U. Use of inflammatory markers for early detection of bacteraemia in patients with febrile neutropenia. Scand J Infect Dis. 2004;36(5):365-71.

26. Giamarellou H, Giamarellos-Bourboulis EJ, Repoussis P, Galani L, Anagnostopoulos N, Grecka P, Lubos D, Aoun M, Athanassiou K, Bouza E, Devigili E, Krcmery V, Menichetti F, Panaretou E, Papageorgiou E, Plachouras D. Potential use of procalcitonin as a diagnostic criterion in febrile neutropenia: experience from a multicentre study. Clin Microbiol Infect. 2004 Jul;10(7):628-33.

27. Jimeno A, Garcia-Velasco A, del Val O, Gonzalez-Billalabeitia E, Hernando S, Hernandez R, Sanchez-Munoz A, Lopez-Martin A, Duran I, Robles L, Cortes-Funes H, PazAres L. Assessment of procalcitonin as a diagnostic and prognostic marker in patients with solid tumors and febrile neutropenia. Cancer. 2004 Jun 1;100(11):2462-9.

28. Moellering RC Jr. Principles of anti-infective therapy. En: Mandell GL, Bennet JE, Dolin R, eds. Principles and practice of Infectious Diseases. 5Ed Philadelphia : Churchill Livingston ; 2000. p. 223-235.

29. Gómez J. Significación clínica de los factores de riesgo en el diagnóstico y tratamiento de las infecciones graves. Enf Inf Microbiol Clin 1999; 17: 379-381.

30. Engels EA, Lau J, Barza M. Efficacy of quinolone prophylaxis in neutropenic cancer patients: a meta-analysis. J Clin Oncol 1998;16:1179-1187

31. Cullen M, Steven N, Billingham L, Gaunt C. Antibacterial prophylaxis after chemotherapy for solid tumors and lymphomas. N Engl J Med. 2005 Sep 8;353(10):988-98.

32. Elting LS, Rubenstein EB, Rolston KV, Bodey GP. Outcomes of bacteremia in patients with cancer and neutropenia: observations from two decades of epidemiological and clinical trials. Clin Infect Dis. 1997 Aug;25(2):247-59.

33. Low-risk febrile neutropenia in a medical oncology unit. Leong DC, Kinlay S, Ackland S, Bonaventura A, Stewart JF. Aust N Z J Med. 1997 Aug;27(4):403-7.

34. Talcott JA, Siegel RD, Finberg R, Goldman L. Risk assessment in cancer patients with fever and neutropenia: a prospective, two-center validation of a prediction rule. $\mathrm{J}$ Clin Oncol. 1992 Feb;10(2):316-22.

35. Miguel A. Sanz. Cefepime plus amikacin versus piperacillin-tazobactam plus amikacin for initial antibiotic therapy in haematology patients with febrile neutropenia: results of an open, randomized, multicentre trial. Journal of Antimicrobial Chemotherapy (2002) 50, 79-88.

36. Liat Vidal, Mical Paul, Itsik Ben dor, Karla Soares-Weiser and Leonard Leibovic. Oral versus intravenous antibiotic treatment for febrile neutropenia in cancer patients: a sys- tematic review and meta-analysis of randomized trials Journal of Antimicrobial Chemotherapy (2004) 54, 29-37

37. Gardembas PM, Desablens B, Sensebe L et al. Home treatment of febrile neutropenia: an empirical oral antibiotic regimen. Ann Oncol 1991;2:485-487.

38. Elting LS, Bodey GP, Keefe BH. Septicemia and shock syndrome due to viridans streptococci: a case-control study of predisposing factors. Clin Infect Dis. 1992 Jun;14(6):1201-7.

39. Tunkel AR, Sepkowitz KA. Infections caused by viridans streptococci in patients with neutropenia. Clin Infect Dis. 2002 Jun 1;34(11):1524-9.

40. Bochud PY, Eggiman P, Calandra T, Van Melle G, Saghafi L, Francioli P. Bacteremia due to viridans streptococcus in neutropenic patients with cancer: clinical spectrum and risk factors. Clin Infect Dis. 1994 Jan;18(1):25-31.

41. Kubesova H, Penka M, Kral Z, Adam Z, Vasova I, Tomiska M, Vorlicek J. Herpes simplex infection as possible etiology for febrile neutropenia and mucositis in patients treated for hematological malignancies. Acta Med Austriaca. 1998;25(2):57-60.

42. Spielberger R, Stiff P, Bensinger W, Gentile T, Weisdorf D, Kewalramani T, Shea T, Yanovich S, Hansen K, Noga S, McCarty J, LeMaistre CF, Sung EC, Blazar BR, Elhardt D, Chen MG, Emmanouilides C. Palifermin for oral mucositis after intensive therapy for hematologic cancers. N Engl J Med. 2004 Dec 16;351(25):2590-8.

43. Milosevic DB. The different level of immunological recovery after chemotherapy in leukemia and lymphoma patients. J Exp Clin Cancer Res. 2001 Dec;20(4):517-22.

44. Muller SA, Herrmann EC Jr, Winkelmann RK. Herpes simplex infections in hematologic malignancies. Am J Med. 1972 Jan;52(1):102-14. Review.

45. Lam MT, Pazin GJ, Armstrong JA, Ho M. Herpes simplex infection in acute myelogenous leukemia and other hematologic malignancies: a prospective study. Cancer. 1981 Nov 15;48(10):2168-71.

46. Anderson H, Scarffe JH, Sutton RN, Hickmott E, Brigden $\mathrm{D}$, Burke C. Oral acyclovir prophylaxis against herpes simplex virus in non-Hodgkin lymphoma and acute lymphoblastic leukaemia patients receiving remission induction chemotherapy. A randomised double blind, placebo controlled trial. Br J Cancer 1984 Jul;50(1):45-9.

47. Bergmann OJ, Mogensen SC, Ellegaard J. Herpes simplex virus and intraoral ulcers in immunocompromised patients with haematologic malignancies. Eur J Clin Microbiol Infect Dis. 1990 Mar;9(3):184-90.

48. Baglin TP, Gray JJ, Marcus RE, Wreghitt TG. Antibiotic resistant fever associated with herpes simplex virus infection in neutropenic patients with haematological malignancy. J Clin Pathol. 1989 Dec;42(12):1255-8.

49. Mackall CL. T-cell immunodeficiency following cytotoxic antineoplastic therapy: a review. Stem Cells. 2000;18(1): 10-8. Review.

50. Strender LE, Blomgren H, Petrini B, Wasserman J, Forsgren M, Norberg R, Baral E, Wallgren A. Immunologic monito- 
ring in breast cancer patients receiving postoperative adjuvant chemotherapy. Cancer. 1981 Nov 1;48(9):1996-2002.

51. Wijermans PW, Gerrits WB, Haak HL. Severe immunodeficiency in patients treated with fludarabine monophosphate. Eur J Haematol. 1993 May;50(5):292-6.

52. Reckzeh B, Merte H, Pfluger KH, Pfab R, Wolf M, Havemann K. Severe lymphocytopenia and interstitial pneumonia in patients treated with paclitaxel and simultaneous radiotherapy for non-small-cell lung cancer.J Clin Oncol. 1996 Apr;14(4):1071-6.

53. Beattie G, Whelan J, Cassidy J, Milne L, Burns S, Leonard R. Herpes simplex virus, Candida albicans and mouth ulcers in neutropenic patients with non-haematological malignancy. Cancer Chemother Pharmacol. 1989;25(1):75-6.

54. Melichar B, Touskova M, Vesely P. Effect of irinotecan on the phenotype of peripheral blood leukocyte populations in patients with metastatic colorectal cancer. Hepatogastroenterology. 2002 Jul-Aug;49(46):967-70.

55. Liat Vidal, Mical Paul, Itsik Ben dor, Karla Soares-Weiser and Leonard Leibovic. Oral versus intravenous antibiotic treatment for febrile neutropenia in cancer patients: a systematic review and meta-analysis of randomized trials Journal of Antimicrobial Chemotherapy 2004;54, 29-37

56. Freifeld A, Marchigiani D, Walsh T, Chanock S, Lewis L, Hiemenz J, Hiemenz S, Hicks JE, Gill V, Steinberg SM, Pizzo PA. A double-blind comparison of empirical oral and intravenous antibiotic therapy for low-risk febrile patients with neutropenia during cancer chemotherapy. N Engl J Med. 1999 Jul 29;341(5):305-11.

57. Rubenstein EB, Rolston K, Benjamin RS, Loewy J, Escalante C, Manzullo E, Hughes P, Moreland B, Fender A, Kennedy K, et al. Outpatient treatment of febrile episodes in low-risk neutropenic patients with cancer. Cancer. 1993 Jun 1;71(11):3640-6.
58. Rubenstein E, Rolston K, Moreland B et al. Ambulatory treatment of febrile episodes in neutropenic patients. Proc Am Soc Clin Oncol 1990;9:321a.

59. Talcott JA, Whalen A, Clark J et al. Home antibiotic therapy for low-risk cancer patients with fever and neutropenia: a pilot study of 30 patients based on a validated prediction rule. J Clin Oncol 1994;12:107-114.

60. Samonis, G., Anaissie, E. J., Kalbakis, K., et al. Therapy for low-risk cancer patients with fever and neutropenia: results of a prospective, randomized trial with cost analysis. In Programs and Abstracts of the Thirty-seventh Interscience Conference on Antimicrobial Agents and Chemotherapy, Atlanta, GA, 1997. Abstract LM51. American Society for Microbiology, Washington, DC, 1997.

61. Innes HE, Smith DB, O'Reilly SM et al. Oral antibiotic administration and early hospital discharge is a safe and effective alternative for treatment of low-risk neutropenic fever: a prospective randomised controlled single centre study. British Journal of Cancer 2003; 89: 43-49.

62. Mullen CA, Buchanan GR. Early hospital discharge of children with cancer treated for fever and neutropenia: identification and management of the low-risk patient. J Clin Oncol 1990;8:1998-2004.

63. Hidalgo M, Hornedo J, Lumbreras C, Trigo JM, Colomer R, Perea S, Gómez C, Ruiz A, García-Carbonero R, Cortés-Funes H. Outpatient therapy with oral ofloxacin for patients with low risk neutropenia and fever: a prospective, randomized clinical trial. Cancer, 1999 Jan 1;85(1):213-9.

64. Escalante CP, Weiser MA, Manzullo E, Benjamin R, Rivera E, Lam T, Ho V, Valdres R, Lee EL, Badrina N, Fernandez S, DeJesus Y, Rolston K. Outcomes of treatment pathways in outpatient treatment of low risk febrile neutropenic cancer patients. Support Care Cancer. 2004 Sep;12(9):657-62. 